\title{
Potential role of a new PEGylated recombinant factor VIII for hemophilia A
}

This article was published in the following Dove Press journal:

Journal of Blood Medicine

20 June 2016

Number of times this article has been viewed

\author{
Tung Thanh Wynn' \\ Burak Gumuscu ${ }^{2,3}$ \\ 'Department of Pediatrics, Division \\ of Pediatric Hematology/Oncology, \\ University of Florida, Gainesville, FL, \\ ${ }^{2}$ Pediatric Hematology-Oncology, Bon \\ Secours Health System, St. Mary's \\ Hospital, Richmond, VA, ${ }^{3}$ Department \\ of Pediatrics, Division of Pediatric \\ Hematology/Oncology, University of \\ Virginia, Charlottesville, VA, USA
}

\begin{abstract}
Hemophilia A, a deficiency in the activity of coagulation factor (F) VIII, is an X-linked bleeding disorder with an approximate incidence of one in 5,000 male infants. Bleeding-related complications often result in greater severity of disease, poor quality of life, surgical interventions for severe joint destruction, and shortened life span. With the availability of plasma-derived and recombinant FVIII products, the benefits of primary prophylaxis were demonstrated and is now the standard of care for patients with severe factor deficiencies. Current hemophilia research is focusing on the creation of new factor replacement therapies with longer half-lives; accessing alternative mechanisms to achieve desired hemostasis and enhance bypassing activity; and limiting the immunogenicity of the protein. PEGylation involves the covalent attachment of polyethylene glycol (PEG) to a protein, peptide, or a small molecule drug. PEG effectively increases the molecular weight and size of the protein by creating a hydrophilic cloud around the molecule. This molecular change may reduce susceptibility of the molecule to proteolytic activity and degradation. It is also believed that PEGylation changes the surface charge of the protein that ultimately interferes with some receptor-mediated clearance processes. The half-life of PEGylated factor is more prolonged when compared to non-PEGylated full-length recombinant FVIII. The dawn of a new era in the care of hemophilia patients is upon us with the release of recombinant FVIII products with extended half-lives, and products with even more extended half-life will become available in a very short time. With all the promise of these new agents, many questions still remain.
\end{abstract}

Keywords: hemophilia A, PEG, extended half-life, factor VIII deficiency

\section{Introduction}

Hemophilia A, a deficiency in the activity of coagulation factor (F) VIII, is an $\mathrm{X}$-linked bleeding disorder with an approximate incidence of one in 5,000 male infants. ${ }^{1,2}$ Absence or reduction of the FVIII protein affects secondary hemostasis, which manifests as induced or spontaneous bleeding depending on the severity of the disease. The severity of hemophilia is classified depending on the patient's baseline plasma level of FVIII. Coagulation factor levels are often expressed as a percentage of factor activity or as international units. One international unit (IU) is the amount of FVIII in $1 \mathrm{~mL}$ pooled plasma. One hundred percent $(100 \mathrm{IU} / \mathrm{dL})$ is the average amount of activity for a person without hemophilia. Severe hemophilia is characterized by a FVIII level of $<1 \%(1 \mathrm{IU} / \mathrm{dL})$. Levels between $1 \%$ and $5 \%(1-5 \mathrm{IU} / \mathrm{dL})$ result in moderate hemophilia, and levels between $5 \%$ and $40 \%$ (5-40 IU/dL) are considered mild hemophilia. While one-half of all hemophilia
Correspondence: Tung Thanh Wynn Department of Pediatrics, Division of Pediatric Hematology/Oncology, University of Florida, PO Box 100298 , 1600 SW Archer Rd, Gainesville, FL 32610 , USA

Tel +13522739120

Fax + I 352294809 I

Email twynn@ufl.edu 
cases have severe deficiency, moderate and mild factor deficiencies correspond to $10 \%$ and $40 \%$ of cases, respectively. ${ }^{3,4}$ The correlation between factor levels and the severity of bleeding is not perfect, but in general, clinical phenotype corresponds to the factor level. The hallmark clinical presentation of severe hemophilia A is spontaneous, traumatic, and excessive soft tissue, muscle, body cavity, and joint bleeding. Annual treatment cost of severe FVIII deficiency is several hundred thousand US dollars and bleeding-related complications often result in greater severity of disease, poor quality of life, surgical interventions for severe joint destruction, and shortened life span. ${ }^{5}$

\section{History of factor, management issues, and strategies}

From the late 1950s and through the 1960s, fresh frozen plasma was the main treatment modality for hemophilia. Each unit of fresh frozen plasma contains only a small amount of FVIII, thus large volumes of intravenously administered fresh frozen plasma were needed to stop bleeding episodes, and patients were usually hospitalized for treatment of joint bleeding. Due to reluctance to be hospitalized, delayed treatment in many adolescents and young adults led to the development of chronic joint disease with deformities. Over the past several decades, plasmaderived FVIII concentrates and recombinant preparations have become the center of successful management of hemophilia A. ${ }^{2,3}$

Modern management began in the early 1970s with the discovery of plasma-derived FVIII concentrates. This homebased replacement therapy allowed early control of spontaneous bleeding and reduced the degree of musculoskeletal damage. This single, life-altering advancement in hemophilia care became a paragon of the successful management and secondary prevention of the most common hereditary coagulation disorder.

However, a major setback for the entire hemophilia community and plasma-derived concentrate replacement therapy came with the discovery of the transmission of potentially deadly blood-borne pathogens through the products. By the early 1980s, human blood, plasma, and these plasma-derived products manufactured from the pooled plasma donated by thousands of people were discovered to be transmitting viruses, including hepatitis B and C, and HIV. After this discovery, and by the late 1980 s, advanced screening methods were developed and purification steps were incorporated into the production of donor-derived concentrates. Safer plasma concentrates of coagulation factors became available, but, unfortunately, by this time, many patients had already been infected and had succumbed to the epidemic. ${ }^{2,3,6}$

Another major breakthrough for hemophilia treatment following on the heels of this tragedy came with the cloning of the FVIII gene in 1984. After extensive clinical trials and by the 1990s, genetically engineered recombinant factors became the mainstay of hemophilia treatment. ${ }^{2,6,7}$ Infusion of FVIII concentrates either with newer purified plasma-derived factors or these recombinant preparations have led to successful management of hemophilia. ${ }^{2}$

With the availability of plasma-derived and recombinant FVIII products, the benefits of primary prophylaxis were demonstrated and they became the standard of care for patients with severe factor deficiencies. ${ }^{8,9}$ The widespread use of prophylactic regimens has improved joint outcome and increased quality of life, ${ }^{9,10}$ but it has also been associated with the development of neutralizing antibodies, or inhibitors, in $\sim 25.9 \%-32 \%$ of these patients. ${ }^{11,12}$ Additional factors contribute to the risk of inhibitor development, including genetic mutation, age, race, and intensity of exposure, and these are confounding factors to the problem. The development of inhibitors is generally considered the most frequent serious adverse event in these patients. Without an effective rise in factor activity following an infusion, patients continue to bleed frequently and severely, and the benefit of prophylaxis is eliminated.

Low levels of inhibitors may be overcome by utilizing higher doses for infusion, but a different strategy is necessary when the inhibitor levels are high. Patients who develop high levels of inhibitors may be managed effectively with bypass agents, such as activated prothrombin complex concentrate (such as FEIBA [factor eight inhibitor bypassing activity]) or activated FVII. These replacement products are extremely effective in the management of acute bleeds and, for some, in the long-term management of bleeds in patients with inhibitors. However, breakthrough bleeding is still common and leads to early joint damage, hemophilic arthropathy, and long-term disability. Most patients who develop inhibitors will therefore be tried on immune tolerance induction (ITI) therapy. They are exposed to high doses of a FVIII product on a frequent basis. In patients who respond, the repeated exposure allows the immune system to tolerize itself to FVIII and recovery of FVIII activity occurs. Once this is achieved, the frequency of exposure is reduced until a return to a standard prophylactic schedule is achieved. Approximately $70 \%$ of patients will be able to tolerize after going through ITI, and the time that is required for tolerization may be several years. ${ }^{13}$ The cost of high doses of factor can also be prohibitive for some patients. ${ }^{14}$ 


\section{Structure, function, and degradation of FVIII}

The human FVIII protein was purified in 1982 by Fay et al, ${ }^{15}$ and was cloned independently and simultaneously by three different groups in 1984. Toole et $\mathrm{al}^{7}$ isolated clones corresponding to the FVIII gene from a human complementary DNA library. Gitschier et $\mathrm{al}^{16}$ and Wood et $\mathrm{al}^{17}$ also cloned and expressed the FVIII gene. With a $186 \mathrm{~kb}$ size, it is still one of the largest described genes.

In 1986, Tantravahi et a $\mathrm{l}^{18}$ concluded that the FVIII gene is positioned in the most distal band (Xq28) of the long arm of the X chromosome. The FVIII gene comprises of 26 exons, which encode for a polypeptide chain of 2,351 amino acids. Once the 19 peptide secretory leader sequence is removed, FVIII has a mature sequence of 2,332 amino acids with the domain structure A1-a1-A2-a2-B-a3-A3-C1-C2. von Willebrand factor (vWF), which noncovalently binds with plasma FVIII, acts as a carrier and protects FVIII from proteolysis and rapid clearance. The domain structure of FVIII is very similar to that of coagulation $\mathrm{FV}^{15-18}$

The in vivo expression site of FVIII has not been definitively determined. Based on mouse studies, FVIII is believed to be produced in sinusoidal endothelial cells, predominantly in the liver. ${ }^{18}$ However, a variety of other tissues are also capable of producing it, including the spleen, lymph nodes, and kidneys. FVIII is produced as an inactive single-chain protein. After posttranslational modifications, the coagulation factor is released into the circulation as a set of heterodimeric proteins. Once released, the FVIII heterodimer interacts with $\mathrm{vWF}$ and forms a noncovalent complex. When the coagulation cascade is triggered, the FVIII heterodimer becomes subject to multiple proteolytic cleavages by thrombin to form activated FVIII (FVIIIa). FVIIIa acts as an essential cofactor for activated FIX in the contact factor pathway (intrinsic coagulation cascade). After conversion into its active form and participation in this FX activating complex, FVIIIa loses its activity quickly. Inactivation and loss of procoagulant function occur through either spontaneous dissociation of A2-a2 or proteolysis of FVIIIa by activated protein C. ${ }^{15-22}$

FVIII is highly sensitive to proteolytic processing after secretion, and only a small fraction of circulating FVIII is in the single-chain form. The majority consists of heavy chains of variable length (consisting of the A1 and A2 domains together with variable lengths of $\mathrm{B}$ domain) linked noncovalently to light chains consisting of the $\mathrm{A} 3, \mathrm{C} 1$, and $\mathrm{C} 2$ domains. Expression of active recombinant FVIII lacks the entire length of the $\mathrm{B}$ domain. This proves that the $\mathrm{B}$ domain is not required for activation of the protein of procoagulant function. ${ }^{15-18}$

Factor replacement therapy is highly effective in controlling bleeding, but has its limitations. The hemostatic action has a finite time for its effectiveness. Therefore, the therapy frequently requires repeat dosing, and the development of anti-FVIII alloantibodies that inhibit the function of plasma-derived and recombinant FVIII occurs in a significant number of patients. The development of these inhibitory anti-FVIII antibodies is a complex and multifactorial process. Our understanding of the pathophysiological mechanisms leading to alloantibodies development has improved over the past few decades. Inhibitors are defined and controlled by both genetic and environmental factors, including immune regulatory cells (eg, dendritic cells, macrophages, and $\mathrm{CD} 4^{+} \mathrm{T}$ cells), cytokines, and other immune regulatory molecules. The understanding of alloantibody development has improved, but it is still not possible to confidently predict the immune response of an individual patient to a given factor or the risk of developing inhibitory alloantibody at the onset of FVIII replacement therapy. ${ }^{2,22-24}$

\section{Current research and therapy}

Current hemophilia research is focusing on the creation of new factor replacement therapies with longer half-lives, accessing alternative mechanisms to achieve desired hemostasis and to enhance bypassing activity, and on limiting the immunogenicity of the protein. The availability of longeracting factor concentrates would reduce the number of infusions during acute bleeding episodes and prolong the time between prophylactic infusions. Among these newer techniques, covalent attachment of FVIII to polyethylene glycol (PEG; PEGylation), fusion to the fragmented crystallizable $(\mathrm{Fc})$ portion of immunoglobulin $\mathrm{G}_{1}\left(\mathrm{IgG}_{1}\right)$ molecule, and fusion to recombinant albumin are most likely to gain approval. $^{25}$

PEGylation involves the covalent attachment of PEG to a protein, peptide, or a small molecule drug. PEG effectively increases the molecular weight and size of the protein by creating a hydrophilic cloud around the molecule. This molecular change may reduce the susceptibility of the molecule to proteolytic activity and degradation. It is also believed that PEGylation changes the surface charge of the protein that ultimately interferes with some receptor-mediated clearance processes. The pharmacokinetic studies of PEG-FVIII conducted in hemophilic mouse models demonstrated that the half-life of PEGylated factor is more than doubled (4.9 hours) when compared to non-PEGylated full-length recombinant FVIII (1.9 hours). ${ }^{22-25}$ 
Table I Comparison of new and emerging factor VIII replacement products

\begin{tabular}{|c|c|c|c|c|c|}
\hline Factor name & Factor characteristic & Half-life (hours) & ABR & Treatment of bleeds & Reference \\
\hline Octocog alfa (Advate $\left.{ }^{\circledR}\right)$ & $\begin{array}{l}\text { "Standard" factor } \\
\text { concentrate, full-length } \\
\text { molecule }\end{array}$ & $8-12$ & 6.3 & $\begin{array}{l}93 \% \text { bleeding episodes } \\
\text { controlled with one or two } \\
\text { doses }\end{array}$ & 40 \\
\hline $\begin{array}{l}\text { Efmoroctocog alfa } \\
\left(\text { Eloctate }^{\circledR}\right)\end{array}$ & $\begin{array}{l}\text { B-domain deleted factor } \\
\text { VIII-Fc fusion protein }\end{array}$ & 19.0 & $\begin{array}{l}\text { Individualized } \\
\text { prophylaxis I.6, } \\
\text { weekly prohy3.6 }\end{array}$ & $\begin{array}{l}97.8 \% \text { bleeds controlled } \\
\text { with one or two doses }\end{array}$ & 27 \\
\hline $\begin{array}{l}\text { Rurioctocog alfa pegol } \\
\text { (BAX 855, Adynovate }^{\circledR} \text { ) }\end{array}$ & $\begin{array}{l}\text { Full-length factor VIII, } \\
20 \text { kDa PEG }\end{array}$ & $14-19.6$ & 1.9 & $\begin{array}{l}95.9 \% \text { bleeds controlled } \\
\text { with one or two doses }\end{array}$ & 29 \\
\hline $\begin{array}{l}\text { Turoctocog alfa pegol } \\
\text { (N8-GP) }\end{array}$ & $\begin{array}{l}\text { B-domain truncated, } \\
40 \text { kDa PEG }\end{array}$ & 19 & 1.3 & $\mathrm{TBD}$ & 30 \\
\hline $\begin{array}{l}\text { Damoctocog alfa pegol } \\
\text { (BAY 94-9027) }\end{array}$ & $\begin{array}{l}\text { B-domain truncated, } \\
60 \mathrm{kDa} \text { PEG }\end{array}$ & 19 & 1.9 & TBD & 31 \\
\hline CSL 627 & $\begin{array}{l}\text { Single-chain B-domain } \\
\text { deleted }\end{array}$ & TBD & TBD & TBD & 28 \\
\hline
\end{tabular}

Abbreviations: ABR, annualized bleeding rate; PEG, polyethylene glycol; TBD, to be determined.

Human IgG-based therapeutics is another approach for the treatment of a variety of conditions, including hemophilia. Fusion of a biologically active protein to the $\mathrm{Fc}$ domain of human IgG facilitates binding of the protein to the neonatal $\mathrm{Fc}$ receptor $(\mathrm{Fc} \mathrm{Rn}) . \mathrm{Fc} \mathrm{Rn}$ is expressed in many cell types throughout life and is constitutively active. Once the Fc-fusion protein binds to $\mathrm{Fc} \mathrm{Rn}$, it becomes protected from lysosomal degradation. When Fc-fusion proteins and IgG are taken up from circulation into cells by nonspecific endocytosis, they interact with $\mathrm{Fc} \mathrm{Rn}$ endosomes and are redirected back to plasma. This naturally occurring recycle pathway prolongs the half-lives of various biologics, including recombinant FVIII.

Albumin and IgG together account for $\sim 80 \%$ of the proteins in plasma. Although biologically unrelated, both these molecules have an extended plasma half-life exceeding 20 days, which makes them a subject of common interest in fusion protein technologies. It has been shown that in animal models of hemophilia B, genetically fusing recombinant FIX to recombinant albumin factor prolongs the half-life, with increased in vivo recovery and equivalent activity when compared to recombinant FIX only. ${ }^{22-25}$ These provide an alternative to the extension of factor half-life that is shown with PEG.

In fact, efmoroctocog-alfa (Eloctate ${ }^{\circledR}$; Biogen, Cambridge, MA, USA), FVIII-Fc fusio protein, was the first approved recombinant factor specifically designed to extend the halflife of conventional FVIII. Studies have shown that the mean half-life $\left(t^{1 / 2}\right)$ is 19.0 hours, which is $1.5-1.7 \times$ longer than conventional factor. There are two reported prophylactic dosing schedules. One schedule utilizes dosing every 3-5 days based on individual pharmokinetics results, and the other utilizes dosing with alternative fixed dose (65 IU/ $\mathrm{kg}$ ) weekly administration. The $\mathrm{Fc}$-fusion protein product shows superior results with an annualized bleeding rate (ABR) of 1.6 bleeding episodes with individualized prophylaxis and 3.6 bleeding episodes with alternative weekly dosing schedule, compared to an on-demand dosing result of 33.6 bleeding episodes. In addition, $45.3 \%$ of patients on individualized prophylaxis and $17.4 \%$ of patients on alternative weekly prophylaxis had no bleeding at all during the study period compared to $0 \%$ in the group receiving on-demand treatment. During the course of the trials, there were 757 episodes of bleeding. Of these, $87.3 \%$ resolved after one dose of treatment and $97.8 \%$ were treated with only one or two doses. ${ }^{26}$ There is no albumin fusion FVIII replacement product currently in widespread development.

CSL627 is another novel approach. It is a single-chain B-domain deleted FVIII that is activated upon thrombin cleavage. In animal models, the half-life of this factor appears to be enhanced. Of significant note is that it appears to have increased affinity for vWF, and there are some indications that it might have decreased immunogenicity. ${ }^{27}$

\section{New and potential PEG extended half-life factors}

Several PEG factor products are currently approved or are in clinical trials (NCT01736475, NCT01458106, NCT01480180, NCT01580293) in addition to the non-PEG products, and these have or will likely gain approval in the near future (Table 1).

Rurioctocog alfa pegol (BAX 855, Adynovate ${ }^{\circledR}$; Baxalta, Bannockburn, IL, USA) is a full-length recombinant FVIII with a $20 \mathrm{kDa}$ branched PEG covalently bound to the B-domain region and has recently been approved for use in the US. Studies on this product report a half-life that is $1.4 \times$ longer than the half-life of a comparable conventional factor. This results in a $t^{1 / 2}$ of $14-19.6$ hours. There is an 
ABR of 1.9 bleeds on prophylaxis, which is a $90 \%$ reduction compared to on-demand therapy. Of the patients receiving prophylaxis, $39.6 \%$ experienced no bleeds while on the prophylactic therapy. Bleeds were treated with either one or two doses $95.9 \%$ of the time, and a median dose of 30.87 $\mathrm{IU} / \mathrm{kg}$ was used with $97 \%$ of patients, reporting either good or excellent results. There were no reports of any inhibitors after exposure to BAX $855 .^{28}$

Turoctocog alfa pegol (N8-GP) is a glycol-PEGylated product. It is a B-domain truncated FVIII with a $40 \mathrm{kDa}$ PEG bound by a unique O-linked glycan on the residual 21 amino acid B-domain region with a half-life of $1.6 \times$ that of a full-length FVIII product (19 hours). Efficacy data reports an ABR of 1.3 on prophylaxis compared to an on-demand dosing rate of 30.9. Treatment of bleeding events while on clinical trials showed N8-GP was equally effective compared to a standard half-life factor. No patients developed inhibitors while on the studies. ${ }^{29}$

BAY 94-9027 is another B-domain truncated FVIII that has a $60 \mathrm{kDa}$ PEG linked by a modified cysteine region on the A3 domain. The half-life of this product is reported to be 19 hours. The ABR is 1.9 when dosed every 5 days and 3.9 when dosed weekly. There have been two hypersensitivity reactions reported but no reports of any patients with inhibitors. Early reports in animal studies suggested that this compound might result in decreased immunogenicity. ${ }^{30}$

\section{Potential benefits of PEGylated products}

There are multiple foreseeable benefits of using a longer-acting PEGylated product compared to those currently available. With the product's longer half-life, patients will require fewer doses to control their disease. Prophylactic dosing decreases from three times a week or every other day to twice a week or every fifth day. Acute bleeds may be managed with one or two doses of factor replacement rather than continuing the treatment over multiple days or weeks, as is now frequently required. Currently, many patients require hospitalization, not only for the threat to life and limb during a bleeding episode but also due to the frequency of the dosing regimens. As a result of these longer-acting products, many more bleeds may be treatable at home. This in turn may lead to reduced costs associated with hospitalization and absence from work and school, as well as increased productivity when recovering from bleeds. ${ }^{31,32}$

The realized promise of a longer half-life in the PEGylated products results in fewer required doses for prophylaxis, potentially decreasing the burden of infusion and increasing compliance to these regimens. ${ }^{27}$ Many patients who have been reluctant to start prophylactic therapy because of the frequent dosing of these regimens may now be more inclined to consider it and move away from a purely ondemand regimen, which is associated with increased bleeding rates and worsened joint outcomes. ${ }^{9}$ Fewer factor infusions in the pediatric population in particular may also decrease the need for central venous line (CVL) access due to better preservation of patient veins. This in turn, even when a CVL is required, reduces both the burden of infectious complications from a CVL and the risk of venous thrombotic events.

Patient noncompliance with a prophylactic regimen is a challenging factor in hemophilia treatment, especially in teenagers and young adults when compared to younger children. Because prophylaxis is the standard of care in the US and other developed nations, a number of patients may have never experienced a significant bleeding episode. Convincing these patients of the continuing benefits of prophylaxis in the absence of a first-hand bleeding episode is often difficult and almost impossible at times. This noncompliance leads to increased complications seen in adulthood. ${ }^{33}$ A prophylactic regimen that uses fewer needle sticks may help in reducing the number of patients who choose to stop their prophylaxis.

FVIII-Fc fusion protein was the first extended half-life product to gain regulatory approval and was met with cautious enthusiasm. It has now been available in the US for more than a year, but not all patients have consistently observed the gain of a prolonged half-life and its benefits; in fact, some patients have not seen any benefit from this product over standard therapy due to its variability. The introduction of one or more PEGylated products may be an alternative for patients who do not benefit from FVIII-Fc fusion protein products. Inversely, patients who may not respond to PEG factor products may still have any alternative with FVIII-Fc fusion product.

\section{Questions to be answered about long-acting factors}

The extended half-life FVIII replacement products are not a cure for hemophilia A, but are tools that can be used to manage and control the disease. Each new treatment option that becomes available gives rise to the question of how to best use the product along with the other tools available to provide the best possible outcome for each patient. With the greater interpatient variability of dosing, a "one-size-fits-all" model to prophylactic dosing no longer appears to be appropriate. The variation in a product's half-life for each patient results in dosing regimens that range from every 3 days to 
once a week. This dosing challenge has resulted in the need for hemophilia treatment centers to develop a new model for individualized dosing.

Thus far, the primary focus has been on the potential for these products in patients with severe deficiency, but patients with mild and moderate deficiencies could also see benefits. These patients have acute bleeding episodes as well. Due to the infrequency of their bleeds, the proficiency with selfintravenous infusion is not as high. Management of these bleeds can be achieved more simply and with fewer doses in these cases. Some patients with moderate deficiency, and even some patients with mild deficiency, can have regular bleeding. In these patients, a prophylactic regimen, perhaps less dose intensive, might help to prevent long-term joint damage and disability much in the same way that prophylaxis has helped patients with severe deficiency.

With limited information available, the questions arise about when and how to start FVIII products in patients not previously exposed to any FVIII products. The SIPPET trial was designed to demonstrate the difference in inhibitor development when an untreated patient is first exposed to a plasma-derived factor when compared to first exposure with a recombinant product. ${ }^{34}$ Although the study has been completed, the data has not been published yet. However, results of the study suggested that there might be some risk of inhibitor development with the use of recombinant therapy in previously untreated patients when compared to plasma-derived factor products. ${ }^{35}$ This data might argue for the use of a plasma-derived product when initiating prophylaxis, especially in those who have additional high risk features, but might not a PEGylated product be equally effective in reducing the risk of inhibitor formation? Could PEGylation prevent exposure of the antigenic sites of the protein and the prolonged exposure result in early tolerization? How does a PEGylated factor affect inhibitor formation in patients with the highest risk of inhibitor development such as those with large mutations or a family history of inhibitors? Does the size of the PEG moiety, which varies between the products, play a role in altering inhibitor development? So far, there have been no reports of any PEG factor replacement products causing inhibitor formation in the initial clinical trials; however, study results from previously untreated patients are yet to be reported. By decreasing the possibility of a patient developing inhibitors, primary prophylaxis may be warranted earlier in the treatment plan of patients with mild-to-moderate hemophilia rather than as secondary prophylaxis.
After the development of inhibitors, is there a role for the use of extended half-life factor for the induction of immune tolerance? Some feel that a plasma-derived product that contains some amount of vWF and other proteins might modulate the immune system to better protect and prolong exposure to FVIII and improve the induction of immune tolerance. ${ }^{36}$ It is reported that tolerance induction with FVIII-Fc protein does occur in hemophilia A mice. ${ }^{37}$ In addition, Groomes et $\mathrm{al}^{38}$ provide the first report of a patient who was able to reduce his factor inhibitor titers in response to an ITI regimen using FVIII-Fc fusion protein product, although full tolerization was not reported. Might not the protection of FVIII by PEGylation also allow a prolonged exposure and possibly improve the likelihood of ITI? In fact, work is underway to explore this potential, but no results from these studies have been published to date.

Are all changes with the introduction of the new products good? We do not know the unintended consequences of the incremental improvement. A poorly measured, but much hoped for, benefit of an extended half-life product is increased patient satisfaction with health care and an increase in the quality of patients' lives. We expect to see fewer missed days from school and work when bleeds and injuries are more easily and better controlled. But does better bleeding control lead to more risk-taking behaviors? Do fewer doses result in the inability or delay in pediatric patients' learning how to self-infuse? What, if any, is the effect of the frequent and continuous exposure to PEG and the potential buildup in the tissues? So far, there is no evidence of tissue accumulation or harm, but what will be the experience after years and decades of use of these products?

\section{Conclusion}

The dawn of a new era in the care of hemophilia patients is upon us with the release of recombinant FVIII products with extended half-lives, and products with even more extended half-life will become available in the very near future. With all the promise of these new agents, many questions still remain. Over the decades, we have learned much about the treatment of hemophilia A. The collective experience gathered, as the care of hemophilia has improved, provides caution and optimism. We will come to learn how to best use these tools and the answers to our questions will come with the new lessons learned.

\section{Acknowledgment}

The authors would like to thank Liz Gengarelly for her careful and thoughtful technical editing of our work. 


\section{Disclosure}

TTW is a study investigator at his site for the development of products sponsored by Baxalta, Bayer, NovoNordisk, and AstraZeneca. BG reports no conflicts of interest in this work.

\section{References}

1. Hoyer LW. Hemophilia A. N Engl J Med. 1994;330:38-45.

2. Mannucci PM, Tuddenham EGD. The hemophilias from royal genes to gene therapy. N Engl J Med. 2001;344:1773-1779.

3. Antonarakis SE, Rossiter JP, Young M, et al. Factor VIII inversions in severe hemophilia A: results from an international consortium. Blood. 1995;86:2206-2212.

4. Paola JD, Montgomery RR, Gill JC, Flood V. Hemophilia and Von Willebrand disease. In: Orkin SH, Fisher D, Ginsburg D, Look AT, Lux SE, Nathan DG, editors. Nathan and Oski's Hematology and Oncology of Infancy and Childhood. 8th ed. Philadelphia, PA: Elsevier; 2015:1028-1039.

5. Ragni MV. Targeting antithrombin to treat hemophilia. $N$ Engl J Med. 2015;373:389-391.

6. Howard TE, Yanover C, Mahlangu J, et al. Haemophilia management: time to get personal? Haemophilia. 2011;17:721-728.

7. Toole JJ, Knopf JL, Wozney JM, et al. Molecular cloning of a cDNA encoding human antihaemophilic factor. Nature. 1984;312:342-347.

8. Medical and Scientific Advisory Council (MASAC) Recommendations Concerning Prophylaxis. Medical bulletin: 193. New York: National Hemophilia Foundation; 1994

9. Manco-Johnson MJ, Abshire TC, Shapiro AD, et al. Prophylaxis versus episodic treatment to prevent joint disease in boys with severe hemophilia. N Engl J Med. 2007;357(6):535-544.

10. Oladapo AO, Epstein JD, Williams E, Ito D, Gringeri A, Valentino LA. Health-related quality of life assessment in haemophilia patients on prophylaxis therapy: a systematic review of results from prospective clinical trials. Haemophilia. 2015;21(5):e344-358.

11. Gouw SC, van den Berg HM, Fischer K, et al. Intensity of factor VIII treatment and inhibitor development in children with severe hemophilia A: the RODIN study. Blood. 2013;121(20):4046-4055.

12. Fischer K, Lassila R, Peyvandi F; EUHASS participants. Inhibitor development in haemophilia according to concentrate. Four-year results from the European Haemophilia Safety Surveillance (EUHASS) project. Thromb Haemost. 2015;113(5):911-1157.

13. Hay CR, DiMichele DM. The principal results of the International Immune Tolerance Study: a randomized dose comparison. Blood. 2012;119(6):1335-1344.

14. Rocino A, Cortesi PA, Scalone L, Mantovani LG, Crea R, Gringeri A. Immune tolerance induction in patients with haemophilia a and inhibitors: effectiveness and cost analysis in an European Cohort (The ITER Study). Haemophilia. 2016;22(1):96-102.

15. Fay PJ, Chavin SI, Schroeder, et al. Purification and characterization of a highly purified human factor VIII consisting of a single type of polypeptide chain. Proc Natl Acad Sci U S A. 1982;79:7200-7204.

16. Gitschier J, Wood WI, Goralka, et al. Characterization of the human factor VIII gene. Nature. 1984;312:326-330.

17. Wood WI, Capon DJ, Simonsen CC, et al. Expression of active human factor VIII from recombinant DNA clones. Nature. 1984;312:330-337.

18. Tantravahi U, Murty VV, Jhanwar SC, et al. Physical mapping of the factor VIII gene proximal to two polymorphic DNA probes in human chromosome band Xq28: implications for factor VIII gene segregation analysis. Cytogenet Cell Genet. 1986;42:75-79.

19. Lenting PJ, Van Mourik J, Mertens K. The life cycle of coagulation factor VIII in view of its structure and function. Blood. 1998;92:3983-3996.

20. Kumaran V, Benten D, Follenzi A, Joseph B, Sarkar R, Gupta S. Transplantation of endothelial cells corrects the phenotype in hemophilia A mice. J Thromb Haemost. 2005;3(9):2022-2031.
21. Follenzi A, Benten D, Novikoff P, Faulkner L, Raut S, Gupta S. Transplanted endothelial cells repopulate the liver endothelium and correct the phenotype of hemophilia A mice. J Clin Invest. 2008;118(3):935-945.

22. Kemball-Cook G, Gomez K. Molecular basis of hemophilia A. In: Lee CA, Berntorp EE, Keith Hoots W, editors. Textbook of Hemophilia. Hoboken, NJ: Wiley Blackwell; 2014:23-31.

23. Gouw SC, Van der Bom JG, Ljung R. Factor VIII products and inhibitor development in severe hemophilia A. N Engl J Med. 2013;368:231-239.

24. Astermark J. FVIII inhibitors: pathogenesis and avoidance. Blood. 2015;125(13):2045-2051.

25. Mahdi AJ, Obaji SG, Collins PW. Role of enhanced half-life factor VIII AND ix in the treatment of haemophilia. Br J Haematol. 2015;169:768-776.

26. Mahlangu J, Powell JS, Ragni MV, et al. Phase 3 study of recombinant factor VIII Fc fusion protein in severe haemophilia A. Blood. 2014;123(3):317-325.

27. Zollner S, Raquet E, Claar P, et al. Non-clinical pharmacokinetics and pharmacodynamics of rVIII-SingleChain, a novel recombinant singlechain factor VIII. Thromb Res. 2014;134(1):125-131.

28. Konkle BA, Stasyshyn O, Chowdary P, et al. Pegylated, full-length, recombinant factor VIII for prophylactic and on-demand treatment of severe hemophilia A. Blood. 2015;126(9):1078-1085.

29. Tiede A, Brand B, Fischer R, et al. Enhancing the pharmacokinetic properties of recombinant factor VIII: first-in-human trial of glycoPEGylated recombinant factor VIII in patients with hemophilia A. J Thromb Haemost. 2013;11(4):670-678.

30. Coyle TE, Reding MT, Lin JC, Michaels LA, Shah A, Powell J. Phase I study of BAY 94-9027, a pegylated B-domain-deleted recombinant factor VIII with an extended half-life, in subjects with hemophilia A. J Thromb Haemost. 2014;12:488-496.

31. Krishnan S, Vietri J, Furlan R, Duncan N. Adherence to prophylaxis is associated with better outcomes in moderate and severe haemophilia: results of a patient survey. Haemophilia. 2015;21(1):64-70.

32. Shapiro AD, Donfield SM, Lynn HS, et al. Defining the impact of hemophilia: the Academic Achievement in Children with Hemophilia Study. Pediatrics. 2001;108(6):E105.

33. Duncan N, Shapiro A, Ye X, Epstein J, Luo MP. Treatment patterns, health-related quality of life and adherence to prophylaxis among haemophilia A patients in the United States. Haemophilia. 2012;18(5): 760-765.

34. Mannucci PM, Gringeri A, Peyvandi F, Santagostino E. Factor VIII products and inhibitor development: the SIPPET study (survey of inhibitors in plasma-product exposed toddlers). Haemophilia. 2007;13(Suppl 5): S65-S68.

35. Peyvandi F, Mannucci PM, Garagiola I. Source of factor VIII replacement (plasmatic or recombinant) and incidence of inhibitory alloantibodies in previously untreated patients with severe hemophilia a: The Multicenter Randomized Sippet Study. Presented at: ASH 57th Annual Meeting Plenary Session; December 6, 2015; Orlando, FL.

36. Oldenburg J, Jiménez-Yuste V, Peiró-Jordán R, Aledort LM, Santagostino E. Primary and rescue immune tolerance induction in children and adults: a multicentre international study with a VWF-containing plasma-derived FVIII concentrate. Haemophilia. 2014;20(1): 83-91.

37. Krishnamoorthy S, Liu T, Drager D, et al. Recombinant factor VIII Fc $(\mathrm{rFVIIIFc})$ fusion protein reduces immunogenicity and induces tolerance in hemophilia A mice. Cell Immunol. 2016;301:30-39.

38. Groomes CL, Gianferante DM, Crouch GD, Parekh DS, Scott DW, Liewu K. Reduction of factor VIII inhibitor titers during immune tolerance induction with recombinant factor VIII-Fc fusion protein. Pediatr Blood Cancer. 2016;63:922-924.

39. Blanchette VS, Shapiro AD, Liesner RJ, et al. Plasma and albuminfree recombinant factor VIII: pharmacokinetics, efficacy and safety in previously treated pediatric patients. J Thromb Haemost. 2008; 6(8):1319-1326. 


\section{Publish your work in this journal}

The Journal of Blood Medicine is an international, peer-reviewed, open access, online journal publishing laboratory, experimental and clinical aspects of all aspect pertaining to blood based medicine including but not limited to: Transfusion Medicine; Blood collection, Donor issues, Transmittable diseases, and Blood banking logistics; Immunohematology; Artificial and alternative blood based therapeutics; Hematology; Biotechnology/nanotechnology of blood related medicine; Legal aspects of blood medicine; Historical perspectives. The manuscript management system is completely online and includes a very quick and fair peer-review system. Visit http://www.dovepress.com/ testimonials.php to read real quotes from published authors. 